This item was submitted to Loughborough's Institutional Repository (https://dspace.lboro.ac.uk/) by the author and is made available under the following Creative Commons Licence conditions.

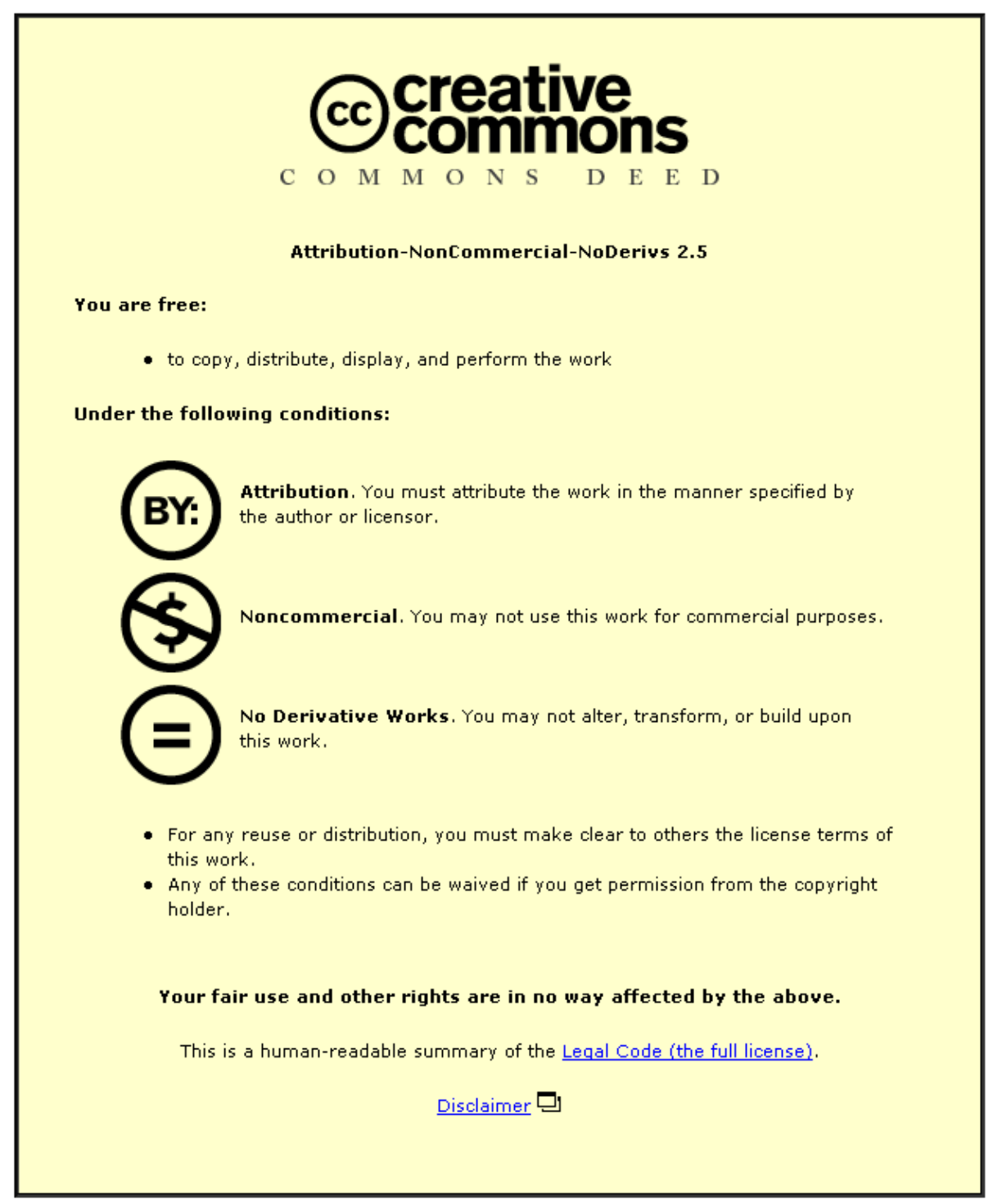

For the full text of this licence, please go to: http://creativecommons.org/licenses/by-nc-nd/2.5/ 


\title{
A perturbation approach to acoustic scattering in dispersions
}

\author{
Valerie J. Pinfield ${ }^{\text {a) }}$ and Malcolm J. W. Povey \\ Procter Department of Food Science, University of Leeds, Leeds, LS2 9JT, United Kingdom
}

(Received 19 October 2005; revised 26 April 2006; accepted 26 April 2006)

\begin{abstract}
Ultrasound spectroscopy has many applications in characterizing dispersions, emulsions, gels, and biomolecules. Interpreting measurements of sound speed and attenuation relies on a theoretical understanding of the relationship between system properties and their effect on sound waves. At its basis is the scattering of a sound wave by a single particle in a suspending medium. The problem has a well-established solution derived by expressing incident and scattered fields in terms of Rayleigh expansions. However, the solution is badly conditioned numerically. By definition, in the long-wavelength limit, the wavelength is much larger than the particle radius, and the scattered fields can then be expressed as perturbation series in the parameter $K a$ (wave number multiplied by particle radius), which is small in this limit. In addition, spherical Bessel and Hankel functions are avoided by using alternative series expansions. In a previous development of this perturbation method, thermal effects had been considered but viscous effects were excluded for simplicity. Here, viscous effects, giving rise to scattered shear waves, are included in the formulation. Accurate numerical correspondence is demonstrated with the established Rayleigh series method for an emulsion. This solution offers a practical computational approach to scattering which can be embodied in acoustic instrumentation. (C) 2006 Acoustical Society of America.
\end{abstract}

[DOI: 10.1121/1.2206512]

PACS number(s): 43.35.Bf [RR]

Pages: $719-732$

\section{INTRODUCTION}

Ultrasound spectroscopy is a noninvasive technique suitable for use with a range of materials, such as emulsions, dispersions, gels, and solutions of biomolecules, with a wide range of particle sizes. In current instrumentation a frequency range of $0.1-200 \mathrm{MHz}$ is used, and particle sizes over a wide range from $10 \mathrm{~nm}$ to $1 \mathrm{~mm}$ can be detected. The measured properties of the ultrasound signal, usually frequency-dependent velocity and attenuation, are related to the characteristics of the system, e.g., the particle size distribution, degree of aggregation, concentration, etc. A substantial review of the application and interpretation of ultrasound measurements can be found in Challis et al. (2005). In order to interpret such ultrasound measurements, it is necessary to relate the properties of the sound wave to the particle size and physical properties of the materials. At the basis of the relationship is the scattering characteristic of a single particle of given properties, and this is the subject of the present work. For an ensemble of such scatterers (such as an emulsion or dispersion), the combined effect on the sound wave is determined by multiple scattering theory.

The long-standing solution to the single-particle scattering problem uses partial wave expansions after Rayleigh (Strutt, 1896), expressing the sound fields as series of spherical harmonics. His solution was later extended for fluid particles by Epstein and Carhart (1953), and for solid particles by Allegra and Hawley (1972). Their solution is referred to as ECAH. The numerical solution of the resulting six boundary equations (for each partial wave order $n$ ) is badly conditioned, causing inaccuracy with the matrix inversion unless a

\footnotetext{
${ }^{a)}$ Electronic mail: v.j.pinfield@ @leeds.ac.uk
}

high degree of machine precision is used. The spherical Bessel functions are also difficult to calculate at large complex arguments. These numerical difficulties are discussed by O'Neill et al. (2001) and Harlen et al.(2001), and such problems have limited the application of the method. The present work addresses these problems in the long-wavelength region, by the development of an alternative method of solution following Kleinman's method (Kleinman, 1965).

In a series of recent publications (Harlen et al., 2001, 2003, Pinfield et al. 2005) the method which is valid in the long-wavelength region has been developed. In this limiting case, the wavelength of the incident sound wave is much larger than the particle radius, so that $K a \ll 1$, where $K$ is the wave number and $a$ the radius of the particles. The sound fields have therefore been expanded as perturbation series in powers of $K a$, after Kleinman (1965). Continuing the application of Kleinman's method, the radiation condition is satisfied by explicitly including exponential spherical soundwave terms of the form $e^{i K r}$, thus avoiding the use of spherical Bessel and Hankel functions. The scattered field is then determined by solving a series of potential problems. The result is a much better conditioned solution to the boundary conditions which simplifies numerical calculation. It may be anticipated that such a method will lead to further developments concerning nonspherical scattering objects and multiple scattering.

The earlier work (Harlen et al., 2001, 2003) applied to limited conditions within the long-wavelength region in which the thermal wavelength was either much larger or much smaller than the particle size. The most recent paper (Pinfield et al., 2005) modified the method to be valid in the entire long-wavelength region, whatever the size of the thermal wavelength. However, viscous effects were neglected in 
all cases. For a viscous medium, shear waves (a transverse wave mode) are also produced at the particle surface. These shear waves were included in the ECAH method, and lead to a significant contribution to attenuation and velocity in some cases. In the work reported here the additional viscous effects are included in the previously documented method. Since the method itself has been detailed in the earlier paper (Pinfield et al., 2005), it is only summarized here, with emphasis on the new contributions resulting from shear effects. In the next section, the principles of sound-wave propagation in fluids are summarized, and the problem of single-particle scattering is introduced. The ECAH forms for the sound waves are shown, together with the alternative perturbation expansion approach. Sections III and IV show how the wave potentials are defined inside and outside the particle, while Sec. V builds the solution by applying boundary conditions. Explicit results are shown for the lowest order coefficients. Section VI demonstrates the numerical agreement between the new method and the ECAH results for $20 \% \mathrm{v} / \mathrm{v}$ n-hexadecane oil-in-water emulsions in which the density difference between the continuous and dispersed phase is significant.

\section{SOUND FIELDS IN A FLUID}

The equations of motion for sound propagation in homogeneous fluids (including continuity, Navier-Stokes, energy) can be simplified by use of velocity potentials, such that

$$
\mathbf{u}=-\nabla \phi+\nabla \times \boldsymbol{\chi},
$$

where $\mathbf{u}$ is the velocity of the fluid, $\phi$ is a scalar potential, and $\boldsymbol{\chi}$ is a vector potential satisfying the condition $\nabla \cdot \boldsymbol{\chi}=0$. When viscous effects are neglected, such as in the earlier development of the method reported here (Pinfield et al., 2005) the vector potential $\chi$ is not included, and only scalar velocity potentials are required. Viscous effects were, however, included in the earlier ECAH method (Epstein and Carhart, 1953; and Allegra and Hawley, 1972).

Substituting Eq. (1) into the equations of motion results in three separate equations, two for scalar potentials and one for the vector potentials. The two scalar potentials correspond to longitudinal propagational and thermal wave modes, while the vector potential represents a transverse shear wave mode. The propagational mode is the "usual" mode by which sound travels in a fluid, while the thermal mode represents heat flow and the shear mode arises from viscous effects. Both the thermal and shear modes are dissipative and therefore highly localized.

The time dependence is defined by a factor $e^{-i \omega t}$, where $\omega$ is the angular frequency, so that each mode satisfies a Helmholtz equation,

$$
\left(\nabla^{2}+K^{2}\right) \varphi=0 \quad\left(\nabla^{2}+L^{2}\right) \psi=0 \quad\left(\nabla^{2}+S^{2}\right) \boldsymbol{\chi}=\mathbf{0} .
$$

The total scalar potential is the sum of the propagational and thermal wave potentials.

The wave numbers, $K$ for the propagational mode, $L$ for the thermal mode, and $S$ for the shear mode, are given to a very good approximation in fluids by

$$
\begin{aligned}
& K=\frac{\omega}{v}\left(1+i \frac{(\gamma-1) \sigma \omega}{2 v^{2}}\right), \\
& L=\left(\frac{\omega}{2 \sigma}\right)^{1 / 2}(1+i), \\
& S=\left(\frac{\omega}{2 \nu}\right)^{1 / 2}(1+i),
\end{aligned}
$$

where $v$ is the speed of sound, $\gamma$ is the ratio of specific heat capacities, $\sigma$ the thermal diffusivity, such that $\sigma=\tau / \rho C_{p}$ where $\tau$ is the thermal conductivity, $\rho$ is the density, and $C_{p}$ is the specific heat capacity at constant pressure, and $\nu$ $=\eta / \rho$ is the kinematic viscosity, where $\eta$ is shear viscosity.

The wave number of the propagational mode is usually given in the form

$$
K=\frac{\omega}{v}+i \alpha,
$$

where $\alpha$ is the attenuation. It is appropriate to use the measured attenuation in (4) rather than the classical result (3) because of absorption effects which are not included in the classical equations.

The temperature fluctuations resulting from the wave modes are

$$
T=\Gamma_{c} \varphi+\Gamma_{t} \psi,
$$

where

$$
\Gamma_{c}=\frac{-i K^{2}(\gamma-1)}{\beta\left(\omega+i \gamma \sigma K^{2}\right)}, \quad \Gamma_{t}=\frac{-i L^{2}(\gamma-1)}{\beta\left(\omega+i \gamma \sigma L^{2}\right)},
$$

where $\beta$ is the thermal expansivity. Subscript $c$ is used to denote the compressional (or propagational) mode and $t$ the thermal mode. The shear mode does not contribute to the temperature fluctuation.

In the absence of viscous effects (Pinfield et al., 2005), only the isotropic pressure needs to be considered, which results in stress terms in proportion to the density of the material. When both inertial and shear stress are included the stress terms are significantly more complicated, and cannot be expressed by a single factor. The relevant forms for the boundary conditions are given in the next section.

A useful thermodynamic relation is

$$
\gamma-1=\frac{v^{2} \beta^{2} T_{0}}{C_{p}},
$$

where $T_{0}$ is the temperature of the system, not the small temperature fluctuations caused by the wave motion.

\section{A. Scattering of sound waves by particles}

In order to determine the ultrasound propagation parameters for a dispersion of particles, we first consider a plane sound wave of angular frequency $\omega$ incident on a spherical particle of radius $a$. By combining the scattering properties of a number of such individual particles, it is possible to estimate the properties of an ensemble of particles. At the surface of the particle, waves of all modes are produced inside and outside the particle. Boundary conditions must be 
satisfied at the particle surface, including the continuity of fluid velocity components, stress components, temperature, and heat flow. Viscous effects introduce requirements on the velocity component $v_{\theta}$ and the stress component $P_{r \theta}$. The axial symmetry of the incident plane wave reduces the vector potential $\chi$ to a single component $\chi_{\psi}$, and for simplicity this will be referred to simply as $\chi$.

In terms of the wave potentials, the boundary conditions are given below. Primed quantities refer to the inside of the particle and $\varphi_{0}$ is the potential of the incident wave.

For the radial component of velocity $v_{r}$,

$$
\begin{aligned}
\frac{\partial}{\partial r}\left(\varphi_{0}+\varphi+\psi\right)-\frac{1}{r \sin \theta} \frac{\partial}{\partial \theta}(\chi \sin \theta) \\
=\frac{\partial}{\partial r}\left(\varphi^{\prime}+\psi^{\prime}\right)-\frac{1}{r \sin \theta} \frac{\partial}{\partial \theta}\left(\chi^{\prime} \sin \theta\right) .
\end{aligned}
$$

For the velocity component $v_{\theta}$,

$$
\frac{\partial}{r \partial \theta}\left(\varphi_{0}+\varphi+\psi\right)+\frac{1}{r} \frac{\partial}{\partial r}(r \chi)=\frac{\partial}{r \partial \theta}\left(\varphi^{\prime}+\psi^{\prime}\right)+\frac{1}{r} \frac{\partial}{\partial r}\left(r \chi^{\prime}\right) .
$$

In the absence of viscous effects, the continuity of stress reduced to a single equation representing the pressure inside and outside the particle. With viscous effects included, the stress components $P_{r r}$ and $P_{r \theta}$ must be continuous across the boundary.

$$
P_{r r}=P_{r r}^{\prime}
$$

and

$$
P_{r \theta}=P_{r \theta}^{\prime} .
$$

The expressions relating the stress components to the wave potentials are as follows: For each compressional wave mode (the propagational and thermal modes)

$$
P_{r r}=\left(i \omega \rho-2 \eta k^{2}\right) \varphi-2 \eta \frac{\partial^{2} \varphi}{\partial r^{2}},
$$

where $k$ is the wave number for the mode,

$$
P_{r \theta}=2 \eta \frac{\partial}{\partial \theta}\left(\frac{\varphi}{r^{2}}-\frac{1}{r} \frac{\partial \varphi}{\partial r}\right) .
$$

Inside the particle, the physical properties for the particle should be used.

For the shear-wave mode, with axial symmetry (see the next section) the stress components are

$$
\begin{aligned}
& P_{r r}=\frac{2 \eta}{\sin \theta} \frac{\partial}{\partial \theta}\left[\sin \theta\left(-\frac{\chi}{r^{2}}+\frac{1}{r} \frac{\partial \chi}{\partial r}\right)\right], \\
& P_{r \theta}=\eta\left[\left(\frac{2 \chi}{r^{2}}-\frac{\partial^{2} \chi}{\partial r^{2}}\right)+\frac{1}{r^{2}} \frac{\partial}{\partial \theta}\left(\frac{1}{\sin \theta} \frac{\partial}{\partial \theta}(\chi \sin \theta)\right)\right],
\end{aligned}
$$

where $\eta$ is the shear viscosity. These equations for stress are appropriate for fluids. Further details on the stress relations are given by Povey (1997, pp. 106-109), and also by Epstein and Carhart (1953) and Allegra and Hawley (1972).
The thermal boundary conditions are unaffected by the shear waves and remain as in the previous work. The temperature continuity equation is

$$
\Gamma_{c} \varphi_{0}+\Gamma_{c} \varphi+\Gamma_{t} \psi=\Gamma_{c}^{\prime} \varphi^{\prime}+\Gamma_{t}^{\prime} \psi^{\prime},
$$

and the heat flux boundary condition

$$
\Gamma_{c} \frac{\partial}{\partial r}\left(\varphi_{0}+\varphi\right)+\Gamma_{t} \frac{\partial \psi}{\partial r}=\hat{\tau}\left(\Gamma_{c}^{\prime} \frac{\partial \varphi^{\prime}}{\partial r}+\Gamma_{t}^{\prime} \frac{\partial \psi^{\prime}}{\partial r}\right),
$$

where $\hat{\tau}=\tau^{\prime} / \tau$ is the ratio of the thermal conductivities in each substance. When evaluating the boundary conditions, the following relations which were listed by Epstein and Carhart (1953) are useful:

$$
\begin{aligned}
& -\frac{d P_{n}(\cos \theta)}{d \theta}=P_{n}^{1}(\cos \theta), \\
& \frac{1}{\sin \theta} \frac{d}{d \theta}\left(\sin \theta P_{n}^{1}(\cos \theta)\right)=n(n+1) P_{n}(\cos \theta) .
\end{aligned}
$$

The objective of the current work is to determine the scattered-wave amplitude for the system of a plane wave and spherical particle. The scattering amplitudes can be combined for an ensemble of particles to calculate the velocity and attenuation of a dispersion. To solve the scattering problem, the new general forms for each wave mode are proposed in Secs. III and IV, which satisfy the appropriate Helmholtz equation given in Sec. II, inside or outside the particle. Section V shows how the solution is constructed, applying the boundary conditions in Sec. V D in order to obtain the solution for scattered-wave amplitudes. Details of the method were given in a previous paper (Pinfield et al., 2005), and are only summarized here. This paper is concerned with the additional effects introduced by the inclusion of viscous effects. First, the scattered waveforms for the ECAH method are shown.

\section{B. ECAH method}

In the ECAH method, the solution potentials were expressed as Rayleigh series, using the spherical harmonic functions. For the radial dependence, the Hankel function $h_{n}$ is used outside the particle, whereas inside the particle the spherical Bessel function $j_{n}$ function is appropriate. The angular dependence is defined by the Legendre polynomials $\left(P_{n}\right)$ and associated Legendre polynomials $\left(P_{n}^{1}\right)$. The vector potential in Epstein and Carhart (1953) used the symbol $A$; here, $\chi$ has been used to avoid confusion with the scattering coefficient $A_{n}$. ECAH took the forms for each wave potential to be as follows:

$$
\begin{aligned}
& \varphi=\sum_{n=0}^{\infty} i^{n}(2 n+1) A_{n} h_{n}(K r) P_{n}(\cos \theta), \\
& \psi=\sum_{n=0}^{\infty} i^{n}(2 n+1) B_{n} h_{n}(\operatorname{Lr}) P_{n}(\cos \theta), \\
& \chi=\sum_{n=1}^{\infty} i^{n}(2 n+1) C_{n} h_{n}(\operatorname{Sr}) P_{n}^{1}(\cos \theta),
\end{aligned}
$$




$$
\begin{aligned}
& \varphi^{\prime}=\sum_{n=0}^{\infty} i^{n}(2 n+1) A_{n}^{\prime} j_{n}\left(K^{\prime} r\right) P_{n}(\cos \theta), \\
& \psi^{\prime}=\sum_{n=0}^{\infty} i^{n}(2 n+1) B_{n}^{\prime} j_{n}\left(L^{\prime} r\right) P_{n}(\cos \theta), \\
& \chi^{\prime}=\sum_{n=1}^{\infty} i^{n}(2 n+1) C_{n}^{\prime} j_{n}\left(S^{\prime} r\right) P_{n}^{1}(\cos \theta) .
\end{aligned}
$$

Similarly, the incident field (a plane wave) can be expressed as

$$
\varphi_{0}=\sum_{n=0}^{\infty} i^{n}(2 n+1) j_{n}(K r) P_{n}(\cos \theta) .
$$

The boundary conditions are applied by evaluating these functions and the appropriate derivatives at the particle surface $r=a$. The resulting set of equations is ill-conditioned numerically, and the Hankel and Bessel functions cause difficulties at some arguments. A full discussion of these difficulties is given by Harlen et al. (2001), showing that under some conditions the matrix becomes very nearly singular, which leads to inaccurate solution if insufficient computational accuracy is used. These problems led us to propose the construction of an alternative form of solution avoiding the use of Bessel functions and more appropriate to numerical solution.

\section{Kleinman method and Poincaré series}

Kleinman showed that a solution to scattering problems in the long-wavelength region $|K a| \ll 1$ could be obtained by expanding the wave potentials as a perturbation series, thus

$$
\phi=\sum_{m=0}^{\infty}(i K a)^{m} \phi_{m},
$$

where $\phi$ is one of the wave potentials. Thus, instead of solving for the total potential $\phi$, each of a set of potentials $\phi_{m}$ must be found. This may appear to be more difficult, but in fact the series converges rapidly, so that a finite number of terms is required for a given accuracy. The solutions for potentials $\phi_{m}$ do not suffer from the numerical instability seen when solving for the overall potentials. Each of the wave potentials is expanded as a series in $i K a$, so that a set of potentials $\phi_{m}$ must be found for each wave type. The principles of the Kleinman method were described in previous work (Harlen et al., 2001, 2003).

\section{SOLUTION FORMS OUTSIDE THE PARTICLE}

In the continuous phase outside the particle, the solutions of the Helmholtz equation must also satisfy the radiation condition according to Colton and Kress (1998, p. 21) and Kleinman (1965). Such solutions take the form

$$
\phi=\frac{e^{i k r}}{r} \widetilde{\phi}=\frac{e^{i k r}}{r} \sum_{l=0}^{\infty} \frac{f_{l}(\theta, \Omega)}{r^{l}},
$$

where $f_{l}$ is the angular dependence (Harlen et al., 2001).
The spherical Hankel function used in the ECAH method is one suitable solution, each $h_{n}(k r)$ including a factor $e^{i k r} / r$ which represents a spherical outgoing wave. However, radiating solutions to the Helmholtz equation are not regular at infinity and it is the exponential part of the function which causes numerical difficulties at large arguments (for the thermal waves). The function $\widetilde{\phi}$ does not suffer from the mathematical difficulties of the overall potential $\phi$. Hence, the radiating terms of the wave potentials outside the particle have been explicitly included so that the boundary equations can work only with the remaining part of the potentials. So, applying Kleinman's method to each mode potential,

$$
\begin{aligned}
& \varphi=e^{i K(r-a)} \tilde{\varphi}, \\
& \psi=e^{i L(r-a)} \tilde{\psi}, \\
& \chi=e^{i S(r-a)} \tilde{\chi} .
\end{aligned}
$$

When applying boundary conditions for a spherical particle at $r=a$ the exponential factors do not contribute. The new potential functions are then expressed as series in $i K a$, using the Kleinman method, thus

$$
(\tilde{\varphi}, \tilde{\psi}, \tilde{\chi})=\sum_{m}(i K a)^{m}\left(\tilde{\varphi}_{m}, \tilde{\psi}_{m}, \tilde{\chi}_{m}\right) .
$$

Now, solution forms must be constructed for the partial potentials $\widetilde{\varphi}_{m}$, etc., which satisfy the Helmholtz equation.

\section{A. Propagational mode}

The partial wave potentials for the propagational mode outside the particle are written as

$$
\tilde{\varphi}_{m}=\sum_{n=0}^{\infty} \sum_{j=0} A_{n m j} \cdot \frac{r^{j}}{a^{j}} \cdot \frac{a^{n+1}}{r^{n+1}} \cdot P_{n}(\cos \theta) .
$$

The full wave potential can be constructed using Eqs. (25) and (22). Substitution into the Helmholtz Eq. (2) leads to the recurrence relation

$$
A_{n, m, j}=-\frac{2(j-1-n)}{j(j-1-2 n)} A_{n, m-1, j-1} \quad \text { for } j \geqslant 1 .
$$

Coefficients for order $m$ are related to those of the previous order $m-1$ for all $j \geqslant 1$. The coefficient for $j=0$ remains to be solved from the boundary equations. The series is finite, terminating at $j=n$, since the recurrence relation shows that the coefficients are zero for $j=n+1$. The series often has even fewer terms, as will be seen when the boundary conditions are applied (Sec. V D).

\section{B. Thermal mode}

The thermal wave potential is based on the spherical Hankel function series expansion (see the Appendix and Pinfield et al., 2005), so that the thermal wave potential takes the form 


$$
\widetilde{\psi}_{m}=\sum_{n=0}^{\infty} \sum_{j=1}^{n+1} B_{n m} \cdot \frac{h_{n j}}{(L r)^{j}} \cdot \frac{1}{S_{h L}} \cdot P_{n}(\cos \theta),
$$

where

$$
S_{h L}=\sum_{j=1}^{n+1} \frac{h_{n j}}{(L a)^{j}}
$$

is a normalizing factor which simplifies the boundary equations for numerical solution. This factor has no zeros, with the parameter $L a$ being of the type $\zeta(1+i)$. The coefficients $h_{n j}$ are defined in the Appendix. The complete wave potential results from combining Eq. (28) with Eqs. (25) and (23).

\section{Shear mode}

In a similar way, the shear mode can also be expressed by the Hankel series expansion; thus

$$
\tilde{\chi}_{m}=\sum_{n=0}^{\infty} \sum_{j=1}^{n+1} C_{n m} \cdot \frac{h_{n j}}{(S r)^{j}} \cdot \frac{1}{S_{h S}} \cdot P_{n}(\cos \theta),
$$

where

$$
S_{h S}=\sum_{j=1}^{n+1} \frac{h_{n j}}{(S a)^{j}} .
$$

The complete wave potential results from combining Eq. (30) with Eqs. (25) and (24).

\section{The incident wave}

The incident plane wave was written in the ECAH method in terms of spherical harmonics, Eq. (19), but it can be expressed as a power series in $(i K a)$ by expanding the spherical Bessel function, thus

$$
\varphi_{0}=\sum_{n=0}^{\infty} \sum_{s=0}^{\infty}(i K a)^{n+2 s}\left(\frac{r}{a}\right)^{n+2 s} F_{n}(s) P_{n}(\cos \theta),
$$

where

$$
F_{n}(s)=\frac{2^{n}(2 n+1)(s+n) !}{s !(2 s+2 n+1) !},
$$

when $n$ and $s$ are non-negative integers ( $F$ is zero otherwise). See the Appendix for the numerical calculation of the function.

\section{SOLUTION FORMS INSIDE THE PARTICLE}

Inside the particle, the solutions must satisfy the Helmholtz equation and be defined at the origin (taken as the center of the particle $r=0$ ). The spherical Bessel function $j_{n}$ is one suitable solution, but evaluating it at large complex arguments causes difficulty, so alternative forms are again sought. The radiation condition is not relevant inside the particle.

\section{A. Propagational mode}

For the propagational mode inside the particle, the solution is defined by the perturbation series expansion

$$
\varphi^{\prime}=\sum_{m}(i K a)^{m} \varphi_{m}^{\prime},
$$

and then expressing the potential as a series in powers of $r$

$$
\varphi_{m}^{\prime}=\sum_{n=0}^{\infty} \sum_{j=0} A_{n m j}^{\prime} \cdot \frac{r^{j}}{a^{j}} \cdot \frac{r^{n}}{a^{n}} \cdot P_{n}(\cos \theta) .
$$

Substituting into the Helmholtz Eq. (2) results in the recurrence relation (36),

$$
\begin{aligned}
& A_{n, m, j}^{\prime}=\frac{\hat{c}}{j(2 n+j+1)} A_{n, m-2, j-2}^{\prime} \quad \text { for } j \geqslant 2, \\
& A_{n, m, j}^{\prime}=0 \quad \text { for } j=1 \text { and all odd values of } j,
\end{aligned}
$$

where

$$
\hat{c}=\frac{K^{\prime 2}}{K^{2}},
$$

which is frequency independent to a very good approximation.

Thus, coefficients for order $m$ are related to those of a previous order $m-2$ for all even coefficients $j \geqslant 2$. The coefficient for $j=0$ remains to be solved from the boundary equations. In this case the limit of the series is determined by the number of nonzero coefficients for the previous order, producing an expanding triangle of coefficients. The solution of the boundary conditions shows that the first nonzero coefficient is for $n=m, j=0$, so that the limit of the series in $j$ would be $j=m-n$, where $m>n$.

\section{B. Thermal mode}

For the thermal wave, a power series expansion of the function $j_{n}$ would be inappropriate, because the argument of the function in the boundary equations could range from a very small value to a very large (complex) value depending on the frequency and radius, even in the long-wavelength region. Instead, the solution is expressed in terms of a sum of an incoming and outgoing wave,

$$
\psi^{\prime}=e^{i L^{\prime}(r-a)} \tilde{\psi}_{+}^{\prime}-e^{-i L^{\prime}(r-a)} \tilde{\psi}_{-}^{\prime} .
$$

Each of the new wave potentials is written as a perturbation series

$$
\left\{\tilde{\psi}_{+}^{\prime}, \tilde{\psi}_{-}^{\prime}\right\}=\sum_{m}(i K a)^{m}\left\{\tilde{\psi}_{+m}^{\prime}, \tilde{\psi}_{-m}^{\prime}\right\},
$$

and each of the terms has the usual angular dependence; thus

$$
\begin{aligned}
& \widetilde{\psi}_{+m}^{\prime}=\sum_{n=0}^{\infty} \sum_{l=1}^{n+1} B_{n m}^{\prime} \cdot e^{2 i L^{\prime} a} \cdot \frac{j_{n l+}}{\left(L^{\prime} r\right)^{l}} \cdot \frac{1}{S_{j L}} \cdot P_{n}(\cos \theta) \\
& \widetilde{\psi}_{-m}^{\prime}=\sum_{n=0}^{\infty} \sum_{l=1}^{n+1} B_{n m}^{\prime} \cdot \frac{j_{n l-}}{\left(L^{\prime} r\right)^{l}} \cdot \frac{1}{S_{j L}} \cdot P_{n}(\cos \theta),
\end{aligned}
$$

where 


$$
S_{j L}=\sum_{l=1}^{n+1} \frac{e^{2 i L^{\prime} a} j_{n l+}-j_{n l-}}{\left(L^{\prime} a\right)^{l}} .
$$

The coefficients $j_{n l+}$ and $j_{n l-}$ are defined in the Appendix. The factor $e^{2 i L^{\prime} a}$ results from the condition that the potential be defined at the origin. The normalizing factor $S_{j L}$ has no zeros, since the zeros of the Bessel function $J_{\nu}(z)$ only occur for real arguments, for orders $\nu>-1$, whereas the parameter $L^{\prime} a$ is complex, with the form $\zeta(1+i)$.

\section{Shear mode}

The shear mode inside the particle is constructed in a similar way to the thermal wave in the previous section; hence, the wave potential can be written

$$
\begin{aligned}
& \chi^{\prime}=e^{i S^{\prime}(r-a)} \tilde{\chi}_{+}-e^{-i S^{\prime}(r-a)} \tilde{\chi}_{-} \\
& \left\{\widetilde{\chi}_{+}^{\prime}, \widetilde{\chi}_{-}^{\prime}\right\}=\sum_{m}(i K a)^{m}\left\{\widetilde{\chi}_{+m}^{\prime}, \widetilde{\chi}_{-m}^{\prime}\right\} .
\end{aligned}
$$

The shear-wave mode has angular dependence defined by the associated Legendre polynomials,

$$
\begin{aligned}
& \widetilde{\chi}_{+m}^{\prime}=\sum_{n=0}^{\infty} \sum_{l=1}^{n+1} C_{n m}^{\prime} \cdot e^{2 i S^{\prime} a} \cdot \frac{j_{n l+}}{\left(S^{\prime} r\right)^{l}} \cdot \frac{1}{S_{j S}} \cdot P_{n}^{1}(\cos \theta) \\
& \widetilde{\chi}_{-m}^{\prime}=\sum_{n=0}^{\infty} \sum_{l=1}^{n+1} C_{n m}^{\prime} \cdot \frac{j_{n l-}}{\left(S^{\prime} r\right)^{l}} \cdot \frac{1}{S_{j S}} \cdot P_{n}^{1}(\cos \theta),
\end{aligned}
$$

where

$$
S_{j S}=\sum_{l=1}^{n+1} \frac{e^{2 i S^{\prime} a} j_{n l+}-j_{n l-}}{\left(S^{\prime} a\right)^{l}} .
$$

The coefficients $j_{n l+}$ and $j_{n l-}$ are defined in the Appendix.

\section{CONSTRUCTION OF THE SOLUTION}

The analytical forms for the wave modes defined in the previous sections must be substituted into the boundary equations in order to determine the unknown coefficients for each mode. Terms in the same power of $i K a$ are matched, as is the angular dependence. Factors appearing in the equations for the thermal and stress terms must therefore be appropriately expressed as powers of $i K a$. These are considered below.

\section{A. Temperature factors}

The temperature changes caused by the different waves were defined in Eq. (6). The relation

$$
\left|\frac{K^{2}}{L^{2}}\right| \approx \frac{\omega \sigma}{v^{2}} \ll 1
$$

is true over a very wide frequency range (up to $\sim 10^{11} \mathrm{~Hz}$ for water at $30{ }^{\circ} \mathrm{C}$ ). Thus, the thermal factors for the propagational mode can be simplified to

$$
\Gamma_{c}=\frac{-i K^{2}(\gamma-1)}{\beta\left(\omega+i \gamma \sigma K^{2}\right)} \approx \frac{K^{2}(\gamma-1)}{\beta \sigma L^{2}}
$$

and

$$
\Gamma_{c}^{\prime}=\frac{-i K^{\prime 2}\left(\gamma^{\prime}-1\right)}{\beta^{\prime}\left(\omega+i \gamma^{\prime} \sigma^{\prime} K^{\prime 2}\right)} \approx \frac{\hat{c} K^{\prime 2}\left(\gamma^{\prime}-1\right)}{\beta^{\prime} \sigma^{\prime} L^{\prime 2}} .
$$

The factors of $(i K a)$ are of interest explicitly, so the thermal factors may be expressed in the form

$$
\Gamma_{c}=(i K a)^{2} g_{c}, \quad \Gamma_{c}^{\prime}=(i K a)^{2} g_{c}^{\prime} .
$$

For the thermal wave mode, the factors can also be simplified and are approximately independent of frequency,

$$
\Gamma_{t}=\frac{-i L^{2}(\gamma-1)}{\beta\left(\omega+i \gamma \sigma L^{2}\right)} \approx-\frac{1}{\beta \sigma},
$$

and similarly in the dispersed phase.

It is also worth noting that the ratio of the temperature factors for the propagational and thermal modes is very small, showing that the temperature changes are dominated by the thermal mode,

$$
\left|\frac{\Gamma_{c}}{\Gamma_{t}}\right| \approx \frac{K^{2}(\gamma-1)}{L^{2}},
$$

which is very small.

\section{B. Stress}

In the absence of viscous effects, the boundary equation for continuity of stress is simply related to the pressure inside and outside the particle surface (Pinfield et al., 2005). The pressure relating to each wave mode was proportional to the frequency and the local density. With the inclusion of viscous stress, the $P_{r r}$ component [Eq. (12)] includes terms in both $(K a)^{2}$ and $(S a)^{2}$ (through the ratio of $\omega \rho / \eta$ ) for the compressional modes (propagational and thermal). The relative magnitude of the contributions to the stress depends on frequency, or equivalently, on the magnitude of $S a$. At low frequencies (known as the viscous limit), the viscous terms are dominant, whereas at high frequency (the inertial limit) the viscous terms are negligible and the motion is governed by the inertia of the particle. The $P_{r \theta}$ boundary contributions are proportional to the shear viscosity.

\section{Definitions}

The application of the boundary conditions leads to some complicated equations, which can be made simpler to read by using some further symbols to define collections of terms.

For the continuous phase

$$
\begin{aligned}
S_{h L} & =\sum_{j=1}^{n+1} \frac{h_{n j}}{(L a)^{j}}, \\
S_{d h L} & =\sum_{j=1}^{n+1} \frac{(i L a-j) h_{n j}}{(L a)^{j}},
\end{aligned}
$$




$$
S_{d 2 h L}=\sum_{j=1}^{n+1} \frac{\left[-L a^{2}-2 i L a j+j(j+1)\right] h_{n j}}{(L a)^{j}},
$$

and similar definitions for the shear wave factors $S_{h S}, S_{d h S}$, $S_{d 2 h S}$, replacing $L a$ by $S a$ in each case. In addition,

$$
S_{d 2 h S S}=\frac{S_{d 2 h S}}{S_{h S}}-\{2-n(n+1)\} .
$$

For the dispersed phase

$$
\begin{aligned}
S_{j L}= & \sum_{l=1}^{n+1} \frac{e^{2 i L^{\prime} a} j_{n l+}-j_{n l-}}{\left(L^{\prime} a\right)^{l}}, \\
S_{d j L}= & \sum_{l=1}^{n+1} \frac{e^{2 i L^{\prime} a}\left(i L^{\prime} a-l\right) j_{n l+}+\left(i L^{\prime} a+l\right) j_{n l-}}{\left(L^{\prime} a\right)^{l}}, \\
S_{d 2 j L}= & \sum_{l=1}^{n+1}\left\{e^{2 i L^{\prime} a}\left[-L^{\prime} a^{2}-2 i L^{\prime} a l+l(l+1)\right] j_{n l+}\right. \\
& \left.+\left[L^{\prime} a^{2}-2 i L^{\prime} a l-l(l+1)\right] j_{n l-}\right\} /\left(L^{\prime} a\right)^{l},
\end{aligned}
$$

and similar definitions for the shear-wave factors $S_{j S}$, etc., replacing $L^{\prime} a$ by $S^{\prime} a$ in each case, with an additional factor

$$
S_{d 2 j S S}=\frac{S_{d 2 j S}}{S_{j S}}-\{2-n(n+1)\} .
$$

For the propagational mode, greater accuracy is achieved (avoiding subtraction of nearly equal terms) by using the recurrence relation (27) to write

$$
\begin{aligned}
S_{j A, m-s} & =\sum_{j=0} A_{n, m-s, j}+\sum_{j=0}^{m-n} j A_{n, m-s+1, j} \\
& =\sum_{j=1}^{m-n}-\frac{j}{(j-2 n)} A_{n, m-s, j}+\delta_{n 0} A_{n, m-s, 0},
\end{aligned}
$$

where $\delta_{n 0}$ is a Kronecker delta such that $\delta_{n j}=1$ for $n=j$ and zero otherwise. Other factors are defined as follows:

$S_{A, m-s}=\sum_{j=0}^{n} A_{n, m-s, j} \quad$ for $s=1,2,3 \quad S_{A, m}=\sum_{j=1}^{n} A_{n, m, j}$,

$S_{j A, m-s}=\sum_{j=1}^{n}-\frac{j}{(j-2 n)} A_{n, m-s, j}+\delta_{n 0} A_{n, m-s, 0} \quad$ for $s=1,2,3$,

$S_{j j A, m-s}=\sum_{j=1}^{n} j A_{n, m-s, j} \quad$ for $s=0,1,2,3$,

$S_{j 2 A, m-s}=\sum_{j=1}^{n} j^{2} A_{n, m-s, j} \quad$ for $s=0,1,2,3$,
$S_{A^{\prime}, m-s}=\sum_{j=0}^{m-n} A_{n, m-s, j}^{\prime} \quad$ for $s=1,2,3 \quad S_{A^{\prime}, m}=\sum_{j=1}^{m-n} A_{n, m, j}^{\prime}$,

$S_{j A^{\prime}, m-s}=\sum_{j=1}^{m-n} j A_{n, m-s, j}^{\prime} \quad$ for $s=0,1,2$,

$S_{j 2 A^{\prime}, m-s}=\sum_{j=1}^{m-n} j^{2} A_{n, m-s, j}^{\prime} \quad$ for $s=0,1,2$.

\section{Boundary conditions}

The boundary conditions [Eqs. (8)-(17)] are applied at the surface of the spherical particle $r=a$. Since both the Legendre polynomials and associated Legendre polynomials of different orders $n$ are independent, the angular dependence in the boundary equations can only be satisfied if applied independently for each order $n$. In other words, there is a set of six boundary equations for each $n$. Each set must be solved independently for the coefficients of that order. The wave potentials have been defined as series in powers of $i K a$, so each boundary equation consists of summations over orders $m$. Terms in powers of $(i K a)$ which may arise from various orders of $m$ are matched on each side of the equation. If each order $m$ is determined in turn, all coefficients for previous orders, e.g., $m-1$, are already known. In addition, the propagational mode coefficients for order $m$ for $j \geqslant 1$ can be calculated from the previous order results [see Eqs. (27) and (36)]. Hence, the boundary equations for the $n, m$ th order include six unknowns,

$$
A_{n m 0}, \quad A_{n m 0}^{\prime}, \quad B_{n m}, \quad B_{n m}^{\prime}, \quad C_{n m}, \quad C_{n m}^{\prime} .
$$

Note that these coefficients do not have the same definition as in the previous work (Pinfield et al., 2005) because of the inclusion of normalization factors which were not previously used, e.g., Eqs. (28) and (29). These normalizing factors help to condition the numerical matrix solution resulting from the boundary equations. The six boundary conditions (8)-(17) in the order $\left\{v_{r}, P_{r r}, v_{\theta}, P_{r \theta}, T\right.$, heat flux $\}$ result in the equations below,

$$
\begin{aligned}
& m F_{n}\left(\frac{m-n}{2}\right)-(n+1)\left[A_{n m 0}+S_{A, m}\right]+S_{j A, m_{-} 1} \\
& \quad+\frac{S_{d h L}}{S_{h L}} B_{n m}-n(n+1) C_{n m} \\
& \quad=n\left[A_{n m 0}^{\prime}+S_{A^{\prime}, m}\right]+S_{j A^{\prime}, m}+\frac{S_{d j L}}{S_{j L}} B_{n m}^{\prime}-n(n+1) C_{n m}^{\prime}, \quad(70) \\
& \left\{(S a)^{2}-2 m(m-1)\right\} F_{n}\left(\frac{m-n}{2}\right)+2 F_{n}\left(\frac{m-n-2}{2}\right) \\
& \quad+\left\{(S a)^{2}-2(n+1)(n+2)\right\}\left[A_{n m 0}+S_{A, m}\right] \\
& \quad-2\left\{-(2 n+3) S_{j j A, m}+S_{j 2 A, m}-2(n+1) S_{A, m-1}+2 S_{j j A, m-1}\right\}
\end{aligned}
$$

V. Pinfield and M. Povey: Acoustic scattering in dispersions 


$$
\begin{aligned}
+ & \left\{(S a)^{2}-2 \frac{S_{d 2 h L}}{S_{h L}}\right\} B_{n m}+2 B_{n m-2} \\
+ & 2 n(n+1)\left\{\frac{S_{d h S}}{S_{h S}}-1\right\} C_{n m} \\
& \left.=\hat{\eta}^{\prime}\left(S^{\prime} a\right)^{2}-2 n(n-1)\right\}\left[A_{n m 0}^{\prime}+S_{A^{\prime}, m}\right] \\
& \left.-2 \hat{\eta}^{\prime}(2 n-1) S_{j A^{\prime}, m}+S_{j 2 A^{\prime}, m}-S_{A^{\prime}, m-2}\right\} \\
+ & \hat{\eta}\left\{\left(S^{\prime} a\right)^{2}-2 \frac{S_{d 2 j L}}{S_{j L}}\right\} B_{n m}^{\prime}+2 \hat{\eta} B_{n m-2}^{\prime} \\
+ & 2 n(n+1) \hat{\eta}\left\{\frac{S_{d j S}}{S_{j S}}-1\right\} C_{n m}^{\prime}, \\
F_{n} & \left(\frac{m-n}{2}\right)+A_{n m 0}+S_{A, m}+B_{n m}-\left\{\frac{S_{d h S}}{S_{h S}}+1\right\} C_{n m} \\
& \left.=A_{n m 0}^{\prime}+S_{A^{\prime}, m}+B_{n m}^{\prime}-\left\{\frac{S_{d j S}}{S_{j S}}+1\right\} C_{n m}^{\prime}\right\}, \\
& \left.+2\left\{\frac{S_{d h L}}{S_{h L}}\right\}\right\} B_{n m}-\frac{S_{d 2 h S S}}{S_{h S}} C_{n m} \\
= & 2(n-1) \hat{\eta}\left[A_{n m 0}^{\prime}+S_{A^{\prime}, m}\right]+2 \hat{\eta} S_{j A^{\prime}, m} \\
2(m+ & 1) F_{n}\left(\frac{m-n}{2}\right)-2(n+2)\left[A_{n m 0}+S_{A, m}\right]+2 S_{j A m-1}
\end{aligned}
$$

These coefficients can be substituted into the other boundary equations, (70)-(73), reducing the system of equations to four equations in four remaining unknown coefficients, $A_{n m 0}$, $A_{n m 0}^{\prime}, C_{n m}, C_{n m}^{\prime}$ (for each order $\left.n, m\right)$.

For the case $n=0$, the boundary conditions in $v_{\theta}$ and $P_{r \theta}$ are identically satisfied, since $P_{0}^{1}(\cos \theta)=0$ [a factor of $P_{n}^{1}$ was canceled to obtain Eqs. (72) and (73)], and the shear wave contributions vanish in the $v_{r}$ and $P_{r r}$ equations. Thus, in this case, Eq. (70) gives

$$
\begin{gathered}
+2 \hat{\eta}\left\{\frac{S_{d j L}}{S_{j L}}-1\right\} B_{n m}^{\prime}-\hat{\eta} \frac{S_{d 2 j S S}}{S_{j S}} C_{n m}^{\prime} \\
g_{c} F_{n}\left(\frac{m-n-2}{2}\right)+g_{c} S_{A, m-2}+\Gamma_{t} B_{n m}=g_{c}^{\prime} S_{A^{\prime}, m-2}+\Gamma_{t}^{\prime} B_{n m}^{\prime} \\
g_{c}(m-2) F_{n}\left(\frac{m-n-2}{2}\right)+g_{c}\left\{S_{j A, m-3}-(n+1) S_{A, m-2}\right\} \\
+\Gamma_{t} \frac{S_{d h L}}{S_{h L}} B_{n m} \\
=\hat{\tau} g_{c}^{\prime}\left\{S_{j A^{\prime}, m-2}+n S_{A^{\prime}, m-2}\right\}+\hat{\tau} \Gamma_{t}^{\prime} \frac{S_{d j L}}{S_{j L}} B_{n m}^{\prime}
\end{gathered}
$$

\section{E. Solution}

The solution proceeds by determining the coefficients for each order $m$ in turn, starting at $m=0$. All coefficients are zero for $m<0$. Nonzero propagational mode coefficients for order $m$ are calculated from the recurrence relations [Eqs. (27) and (36)]. The two thermal boundary conditions, Eqs. (74) and (75), for the $n, m$ th order are unchanged by the addition of viscous effects. As before, since these equations include only the unknown thermal coefficients, other terms being from previous orders $m-2$ and $m-3$, Eqs. (74) and (75) can be solved for the thermal coefficients $B_{n m}$ and $B_{n m}^{\prime}$ as follows:

$$
\begin{aligned}
B_{n m}= & {\left[-g_{c}\left(\hat{\tau} \frac{S_{d j L}}{S_{j L}}-(m-2)\right) F_{n}\left(\frac{m-n-2}{2}\right)-g_{c}\left(\hat{\tau} \frac{S_{d j L}}{S_{j L}}+(n+1)\right) S_{A, m-2}+g_{c} S_{j A, m-3}\right.} \\
& \left.+\hat{\tau}_{c}^{\prime}\left\{\left(\frac{S_{d j L}}{S_{j L}}-n\right) S_{A^{\prime}, m-2}-S_{j A^{\prime}, m-2}\right\}\right] / \Gamma_{t}\left(\hat{\tau} \frac{S_{d j L}}{S_{j L}}-\frac{S_{d h L}}{S_{h L}}\right), \\
B_{n m}^{\prime}= & {\left[-g_{c}\left(\frac{S_{d h L}}{S_{h L}}-(m-2)\right) F_{n}\left(\frac{m-n-2}{2}\right)-g_{c}\left(\frac{S_{d h L}}{S_{h L}}+(n+1)\right) S_{A, m-2}+g_{c} S_{j A, m-3}\right.} \\
& \left.+g_{c}^{\prime}\left\{\left(\frac{S_{d h L}}{S_{h L}}-n \hat{\tau}\right) S_{A^{\prime}, m-2}-\hat{\tau} S_{j A^{\prime}, m-2}\right\}\right] / \Gamma_{t}^{\prime}\left(\hat{\tau} \frac{S_{d j L}}{S_{j L}}-\frac{S_{d h L}}{S_{h L}}\right) .
\end{aligned}
$$

Viscous effects are seen only indirectly in the $A_{0 m 0}$ coefficient through the coefficient from the previous order and the $A_{0 m j}^{\prime}$ coefficients. No viscous contribution is present for the first nonzero coefficient, for $m=2$. The propagational coeffi-

$$
A_{0 m 0}=m F_{0}\left(\frac{m}{2}\right)+A_{0, m-1,0}-S_{j A^{\prime} m}+\frac{S_{d h L}}{S_{h L}} B_{0 m}-\frac{S_{d j L}}{S_{j L}} B_{0 m}^{\prime} .
$$


cient inside the particle is more complicated, resulting from the contributions to stress from both inertial and viscous effects as follows:

$$
\begin{aligned}
\hat{\rho}(S a)^{2} A_{0 m 0}^{\prime}= & (m+1)\left\{(S a)^{2}-2 m\right\} F_{0}\left(\frac{m}{2}\right)+2 F_{0}\left(\frac{m-2}{2}\right) \\
& +(S a)^{2}\left\{A_{0, m-1,0}-\hat{\rho} S_{A^{\prime}, m}\right\} \\
& -\left\{(S a)^{2}+2(\hat{\eta}-2)\right\} S_{j A^{\prime}, m}+2 \hat{\eta}\left(S_{j 2 A^{\prime} m}\right. \\
& \left.-S_{A^{\prime}, m-2}\right)+\left\{(S a)^{2}\left(\frac{S_{d h L}}{S_{h L}}+1\right)\right. \\
& \left.-2\left(\frac{S_{d 2 h L}}{S_{h L}}+2 \frac{S_{d h L}}{S_{h L}}\right)\right\} B_{0 m}+2 B_{0, m-2} \\
& -\left\{(S a)^{2}\left(\frac{S_{d j L}}{S_{j L}}+\hat{\rho}\right)-2\left(2 \frac{S_{d j L}}{S_{j L}}+\hat{\eta} \frac{S_{d 2 j L}}{S_{j L}}\right)\right\} B_{0 m}^{\prime} \\
& -2 \hat{\eta} B_{0, m-2}^{\prime},
\end{aligned}
$$

using the relation

$$
\hat{\eta}\left(S^{\prime} a\right)^{2}=\hat{\rho}(S a)^{2}
$$

which arises from the definition of the shear wave numbers [Eq. (3)], where

$$
\hat{\eta}=\eta^{\prime} / \eta \text { and } \hat{\rho}=\rho^{\prime} / \rho \text {. }
$$

For higher orders $n>0$ the four boundary equations (70)-(73) for order $n, m$, together with the solutions (76) and (77), can be solved by a matrix equation for the remaining unknown coefficients $A_{n m 0}, A_{n m 0}^{\prime}, C_{n m}, C_{n m}^{\prime}$. An analytical result could be obtained, but it is complicated and is only helpful for the lowest orders in $m$ when further simplifications are possible. The matrix equation is as follows:

$$
\begin{aligned}
& \underline{L}=\left\{\begin{array}{cccc}
n+1 & n(n+1) & n & -n(n+1) \\
(S a)^{2}-2(n+1)(n+2) & 2 n(n+1)\left(\frac{S_{d h S}}{S_{h S}}-1\right) & \left.-\hat{\eta}_{\eta}\left(S^{\prime} a\right)^{2}-2 n(n-1)\right\} & -2 \hat{\eta} n(n+1)\left(\frac{S_{d j S}}{S_{j S}}-1\right) \\
1 & -\left(\frac{S_{d h S}}{S_{h S}}+1\right) & -1 & \left(\frac{S_{d j S}}{S_{j S}}+1\right) \\
-2(n+2) & -\frac{S_{d 2 h S S}}{S_{h S}} & -2 \hat{\eta}(n-1) & \hat{\eta} \frac{S_{d 2 j S S}}{S_{j S}}
\end{array}\right\},
\end{aligned}
$$

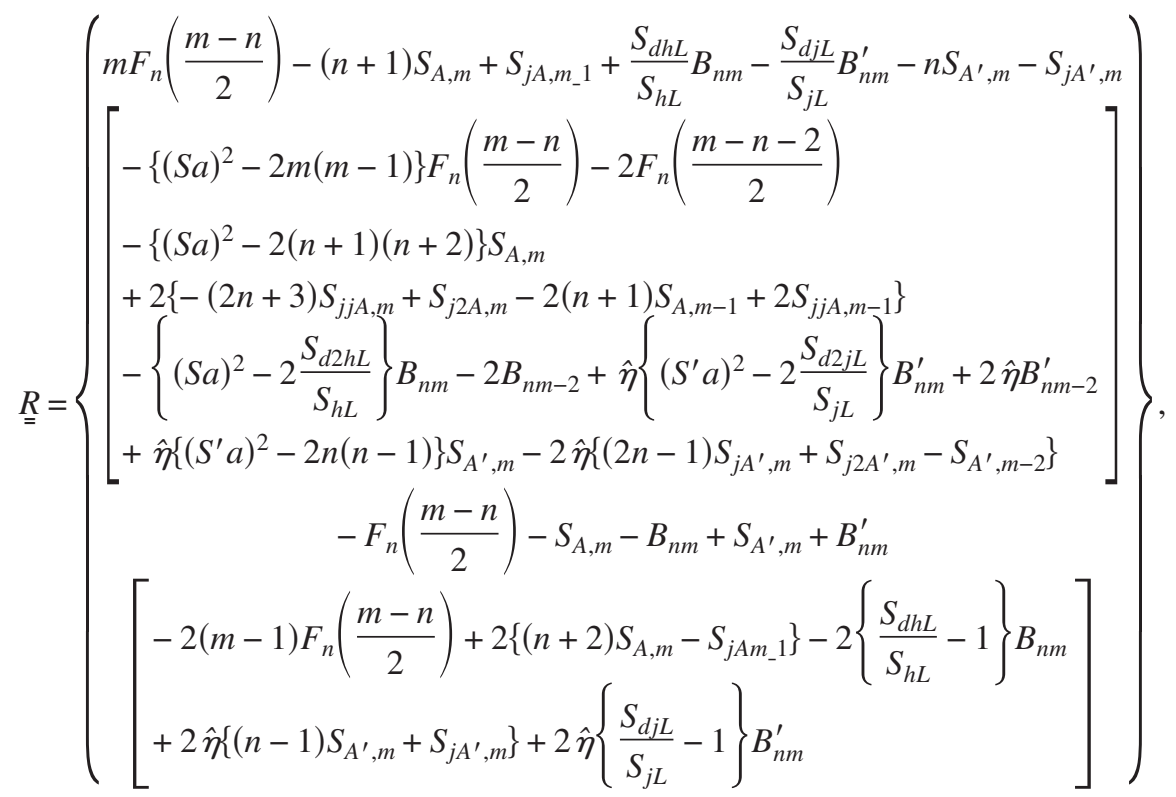




$$
\underline{L}\left\{\begin{array}{c}
A_{n m 0} \\
C_{n m} \\
A_{n m 0}^{\prime} \\
C_{n m}^{\prime}
\end{array}\right\}=\underline{\underline{R}} .
$$

The matrix equation is easily solved by numerical inversion. It is considerably better conditioned than the ECAH matrix equation as a result of the new formulation. Once the coefficients for the order $n, m$ have been found, the process is repeated for the next order $m$, using the recurrence relations, then calculating the thermal coefficients, then solving the matrix equation as before. The complete calculation for all required orders $m$ is repeated for each order $n$, corresponding to the partial wave order (the spherical harmonics).

By examining the boundary equations it is possible to deduce some general features of the solution. Since the lowest order (in $m$ ) contribution from the incident field for an order $n$ is for $m=n$ [see Eq. (82)], the first nonzero coefficient will also be for $m=n$ for the $n$ th-order partial wave. The power series expansion of the incident field [Eq. (32)] shows that the leading term for the $n$th order is indeed $(i K a)^{n}$, resulting in a corresponding factor in the scattered field. The thermal fields [see Eqs. (76) and (77)] are, however, two orders smaller, with a leading term of $(i \mathrm{Ka})^{n+2}$.

If the first nonzero term for the propagational mode coefficient $A_{n m 0}$ is for $m=n$, then the application of the recurrence relation [Eq. (27)] shows that at order $m$, there will be nonzero coefficients for $j \leqslant m-n$ for $m>n$. However, the recurrence relation terminates the series at $j=n$. So, for each order $n$ the maximum number of coefficients for the order $m$ is

$$
j_{\max }= \begin{cases}m-n & \text { for } n \leqslant m \leqslant 2 n \\ n & \text { for } m>2 n .\end{cases}
$$

Hence, there are nonzero coefficients for $j=n$ for all orders $m \geqslant 2 n$. This is relevant in the far field as seen in the next section.

\section{F. Multiple scattering}

In practice, ultrasound measurements are made for a system or ensemble of particles in a dispersion. Usually, the ultrasound velocity (or speed) and/or attenuation is measured, often over a range of frequencies. To relate the singleparticle scattering properties derived in the previous sections to the sound speed and attenuation in a dispersion, a multiple scattering theory is used. Such a theory evaluates the wave number of the dispersed system from the scattering coefficients of the individual particles.

In general, a limiting far-field solution can be written in the form

$$
\varphi \rightarrow \frac{e^{i K r}}{r} f(\theta),
$$

where the scattering amplitude $f(\theta)$ is written in terms of angular-dependent Legendre polynomials; thus

$$
f(\theta)=\frac{1}{i K} \sum_{n=0}^{\infty}(2 n+1) T_{n} P_{n}(\cos \theta),
$$

where $T_{n}$ is an element of the scattering matrix. These parameters are defined in the work of Waterman and Truell (1961), although other workers use slightly different definitions. The parameters can be related to the scattering amplitudes used in the previous sections by comparing Eqs. (85) and (86) with the solutions for the propagational mode, Eqs. (22), (25), and (26). Only coefficients for which $j=n$ contribute in the far field (leading to the $1 / r$ dependence), and since the first nonzero coefficient for $j=n$ is for $m=2 n$ (see Sec. $\mathrm{V} \mathrm{E}$ ), the far-field coefficient $T_{n}$ is related to the coefficient $A_{n m j}$ by

$$
T_{n}=\frac{e^{-i K a}}{(2 n+1)} \sum_{m=2 n}^{\infty}(i K a)^{m+1} A_{n m n} .
$$

The multiple scattering result for the wave number of the dispersion, $B$ (Waterman and Truell, 1961, and Fikioris and Waterman, 1964; Lloyd and Berry, 1967)

$$
\begin{aligned}
\left(\frac{B}{K}\right)^{2}= & 1+\frac{3 \phi}{K^{2} a^{3}} f(0)+\frac{9 \phi^{2}}{4 K^{4} a^{6}}\left(f^{2}(\pi)-f^{2}(0)\right. \\
& \left.-\int_{0}^{\pi} d \theta \frac{1}{\sin (\theta / 2)}\left(\frac{d}{d \theta} f^{2}(\theta)\right)\right),
\end{aligned}
$$

which to second order gives

$$
\begin{aligned}
\left(\frac{B}{K}\right)^{2}= & 1-\frac{3 i \phi}{K^{3} a^{3}} \sum_{n=0}(2 n+1) T_{n}-\frac{27 \phi^{2}}{K^{6} a^{6}}\left(T_{0} T_{1}+\frac{10}{3} T_{0} T_{2}\right. \\
& \left.+2 T_{1}^{2}+11 T_{1} T_{2}+\frac{230}{21} T_{2}^{2}+\cdots\right)
\end{aligned}
$$

where multiple scattering has been included up to $n=2$. Note that here the symbol $\phi$ refers to the volume fraction of the dispersed particles.

Since the wave number is related to the velocity and attenuation by Eq. (4), the dominant contributions to velocity and attenuation scale as

$$
\frac{T_{n}}{(K a)^{3}}
$$

[Equation (89)], so the leading term corresponds to $(K a)^{2 n-2}$. If velocity and attenuation are to be found to order $(K a)^{p}$, spherical harmonics is required up to order

$$
n=\left[\frac{(p+2)}{2}\right]
$$

(where [] refers to the integer part), and orders up to

$$
m=p+2
$$

must be included. As an example, to obtain results for attenuation to order $(K a)^{2}$, i.e., $p=2$, all coefficients up to and including $m=4$ and $n=2$ must be calculated. Note that this is only applicable for $p>0$. The leading-order terms for $p=0$ result from $n=0$ and $n=1$ up to order $m=2$. 


\section{G. Explicit solutions for low orders}

Analytical results have been determined for the coefficients which contribute to attenuation up to order $(\mathrm{Ka})^{0}$. Those coefficients are $A_{020}$ and $A_{121}$. The corresponding farfield coefficients are

$$
T_{0}=e^{-i K a}(i K a)^{3} A_{020}, \quad T_{1}=\frac{e^{-i K a}}{3}(i K a)^{3} A_{121}, \quad T_{2}=0 .
$$

Substituting these coefficients into the equation for the wave number of the dispersion, $B$ [Equation (89)] gives

$$
\begin{aligned}
\left(\frac{B}{K}\right)^{2}= & 1-3 \phi e^{-i K a}\left(A_{020}+A_{121}\right) \\
& +3 \phi^{2} e^{-2 i K a}\left(3 A_{020} A_{121}+2 A_{121}^{2}\right) .
\end{aligned}
$$

Higher order terms can be obtained by numerical inversion of the matrix form of the boundary equations as detailed in the earlier section. Here, the analytical forms of the two leading-order coefficients are determined. The process is given in detail by Pinfield et al. (2005) and only the changes introduced by including viscous effects are noted here.
The $A_{020}$ coefficient is unchanged by the additional viscous effects, since it relates to a spherical partial wave and is dominated by the difference in compressibility and thermal properties. Thus,

$$
A_{020}=\frac{(\hat{\rho}-\hat{c})}{3 \hat{\rho}}+\frac{\left(g_{c}^{\prime}-\hat{\rho} g_{c}\right)\left(\hat{\tau} \Gamma_{t}^{\prime}-\Gamma_{t}\right) S_{J} S_{H}}{\hat{\rho} \Gamma_{t} \Gamma_{t}^{\prime}\left(\hat{\tau} S_{J}-S_{H}\right)},
$$

where

$$
\begin{aligned}
& S_{H}=\frac{S_{d h L}}{S_{h L}}=(i L a-1) \\
& S_{J}=\frac{S_{d j L}}{S_{j L}}=\frac{i L^{\prime} a\left(e^{2 i L^{\prime} a}+1\right)}{\left(e^{2 i L^{\prime} a}-1\right)}-1 .
\end{aligned}
$$

The viscous contribution does affect higher orders in $m$ for $n=0$ through the stress contributions. The propagational mode coefficient $A_{020}^{\prime}$ inside the particle is, however, affected by viscous stress.

For the first-order partial wave $n=1$, the far-field coefficient results from $A_{121}$ which is itself related by the recurrence relation [Eq. (27)] to $A_{110}$ as follows:

$$
A_{121}=-A_{110} \text {, }
$$

where the boundary equations lead to the result

$$
\begin{aligned}
& A_{110}=(\hat{\rho}-1)(S a)^{2} /\left\{(2 \hat{\rho}+1)(S a)^{2}-12+\frac{6\left[\left(\hat{\eta} S_{j 2 S}-2 S_{j 1 S}\right)\left(2 S_{h 1 S}+\hat{\rho}(S a)^{2}\right)-\left(S_{h 2 S}-2 S_{h 1 S}\right)\left(2 \hat{\eta} S_{j 1 S}+\hat{\rho}(S a)^{2}\right)\right]}{\left(\hat{\eta} S_{h 1 S} S_{j 2 S}-S_{h 2 S} S_{j 1 S}\right)}\right\}, \\
& S_{h 1 S}=\frac{S_{d h S}}{S_{h S}}-1=\frac{\left((S a)^{2}+3 i S a-3\right)}{(1-i S a)}, \\
& S_{h 2 S}=\frac{S_{d 2 h S S}}{S_{h S}}=\frac{\left(-i(S a)^{3}+3(S a)^{2}+6 i S a-6\right)}{(1-i S a)}, \\
& S_{j 1 S}=\frac{S_{d j S}}{S_{j S}}-1=\frac{\left(\left(S^{\prime} a\right)^{2} e_{-,+}+3 i S^{\prime} a-3 e_{-,+}\right)}{\left(-i S^{\prime} a+e_{-,+}\right)}, \\
& S_{j 2 S}=\frac{S_{d 2 j S S}}{S_{j S}}=\frac{\left(-i\left(S^{\prime} a\right)^{3}+3\left(S^{\prime} a\right)^{2} e_{-,+}+6 i S^{\prime} a-6 e_{-,+}\right)}{\left(-i S^{\prime} a+e_{-,+}\right)}, \\
& e_{-,+}=\frac{\left(e^{2 i S^{\prime} a}-1\right)}{\left(e^{2 i S^{\prime} a}+1\right)} .
\end{aligned}
$$

If only the first term in the denominator of Eq. (96) is used, the result reduces to that shown in the previous work (Pinfield et al., 2005) in which the coefficient relates only to the density difference between the two phases. In that case, viscous effects were neglected so that only the effect of inertia is present in the $n=1$ (dipole) contribution. Here, the coefficient shows a contribution from both inertial and viscous effects, the relative magnitude of which is determined by the parameter $S a$. For a given particle size, at low frequency (small $\mathrm{Sa}$ ) the viscous effects dominate, whereas at high frequency (large $S a$ ) the inertial terms are most important and the density difference becomes the only relevant parameter.

The ECAH method is not limited to the long-wavelength region in which $K a \ll 1$, although the numerical calculation causes some difficulties. However, Epstein and Carhart (1953) did determine analytical solutions for the $n=0$ and $n=1$ coefficients which are valid in the limit $K a \ll 1$ by considering the relative magnitude of the terms in the solution, and taking limiting forms of the functions. Our own solutions are equivalent to expanding the ECAH limiting solutions as series in powers of $K a$ and taking the first few terms, 
TABLE I. Physical properties of $n$-hexadecane in water with $1 \%$ Tween 20 at $20{ }^{\circ} \mathrm{C}$ (McClements and Coupland, 1996).

\begin{tabular}{|c|c|c|}
\hline & Water $+1 \%$ Tween 20 & n-hexadecane \\
\hline Ultrasound velocity $/ \mathrm{m} \mathrm{s}^{-1}$ & 1485.5 & 1357.9 \\
\hline Density $/ \mathrm{kg} \mathrm{m}^{-3}$ & 999.5 & 773 \\
\hline Thermal expansivity/K $\mathrm{K}^{-1}$ & 0.00022 & 0.00091 \\
\hline Viscosity/Pa s & 0.00111 & 0.00334 \\
\hline Specific heat capacity $/ \mathrm{J} \mathrm{kg}^{-1} \mathrm{~K}^{-1}$ & 4182 & 2093 \\
\hline Thermal conductivity/W $\mathrm{m}^{-1} \mathrm{~K}^{-1}$ & 0.59 & 0.14 \\
\hline Attenuation exponent $p$ & 2 & 2 \\
\hline Attenuation factor/ $\mathrm{Np} \mathrm{m}^{-1} \mathrm{MHz}^{-p}$ & 0.025 & 0.101 \\
\hline
\end{tabular}

corresponding to the first orders in $m$. A full analytical comparison has not been carried out, but the numerical results shown in the next section show that the correspondence is accurate.

\section{NUMERICAL RESULTS}

Numerical calculations have been carried out in MATLAB ${ }^{\circledR}$ for a model system of $20 \%$ (by volume) $n$-hexadecane in water at $20^{\circ} \mathrm{C}$ with a particle diameter of $1 \mu \mathrm{m}$. The physical properties of the two components are given in Table I. The complete range of thermal and shear effects can be seen within the long-wavelength limit. Figures 1 and 2 show the velocity and attenuation as a function of frequency (by the parameter $\mathrm{Ka}$ ) calculated by the ECAH method and the theoretical results presented here. Also shown are the results when viscous effects are neglected. Orders up to $n=2$ and $m=4$ were included, corresponding to effects in velocity and attenuation up to order $(K a)^{2}$. Also shown are the results when shear effects are neglected.

The results for velocity and attenuation match closely those determined using the ECAH method up to around $\mathrm{Ka}$ $\sim 0.1$ as shown. Values of $|L a|$ and $|S a|$ are also shown on the plots, demonstrating that the most significant gradient in velocity and magnitude of attenuation occurs when either parameter is of order unity. At the limiting values of very small or very large $|L a|$ or $|S a|$ the velocity changes only slowly with frequency, and the attenuation is much lower. When $|S a| \approx 1$ both inertial and viscous effects make a significant

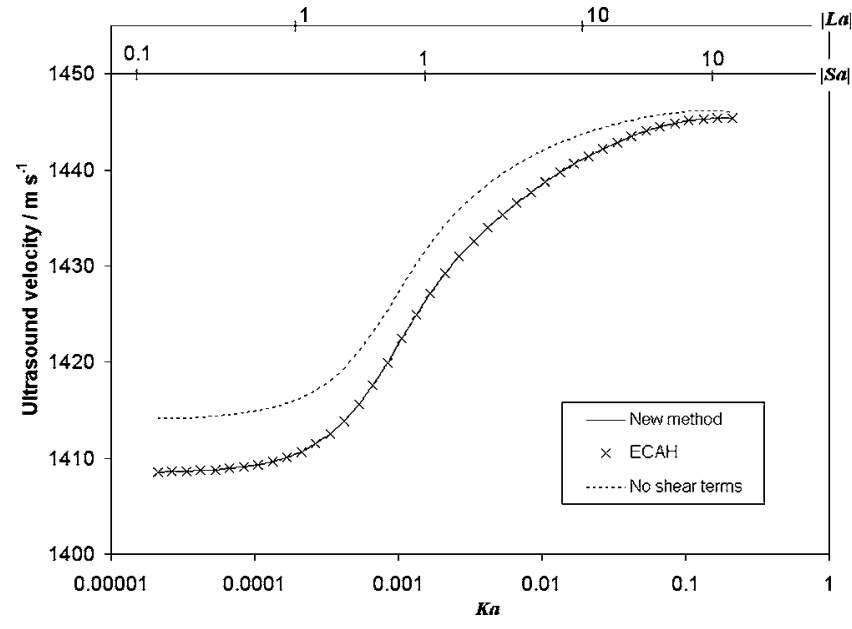

FIG. 1. Ultrasound velocity as a function of the parameter Ka for $20 \%$ hexadecane in water (with $1 \%$ Tween20) at $20^{\circ} \mathrm{C}$ with a particle diameter of $1 \mu \mathrm{m}$. The new method is compared with the previous ECAH method (Epstein and Carhart, 1953) and in the absence of viscous/shear effects. The thermal parameter $|L a|$ and shear parameter $|S a|$ are shown above the plot. The parameters for the dispersed phase are $\left|L^{\prime} a\right|=1.28|L a|,\left|S^{\prime} a\right|=0.52|S a|$, and $K^{\prime} a=1.09 K a$.

contribution and the attenuation is relatively high. Similarly the thermal scattering is greatest when the thermal wavelength is of similar order to the particle size $(|L a| \approx 1)$. In the case of water, $|L a|$ and $|S a|$ are of similar order to each other, causing both visco-inertial and thermal scattering to be significant in overlapping frequency ranges.

For the hexadecane in water system, shear effects make a significant contribution to the ultrasound velocity and attenuation, as can be seen in Figs. 1 and 2. In this case, the parameter $|S a|$ is not applicable, since it tends to infinity, as the viscosity tends to zero. In the previous work (Pinfield et al., 2005) a reasonable agreement was found with the ECAH method in spite of the neglect of viscous effects in that work. The system used for calculations in that case was sunflower oil in water emulsion, for which the density difference between the oil and water is small. The contribution of the visco-inertial scattering to velocity and attenuation is then much smaller. In the present case of a hexadecane in water dispersion, the density difference is much greater, and the visco-inertial effects are more significant.

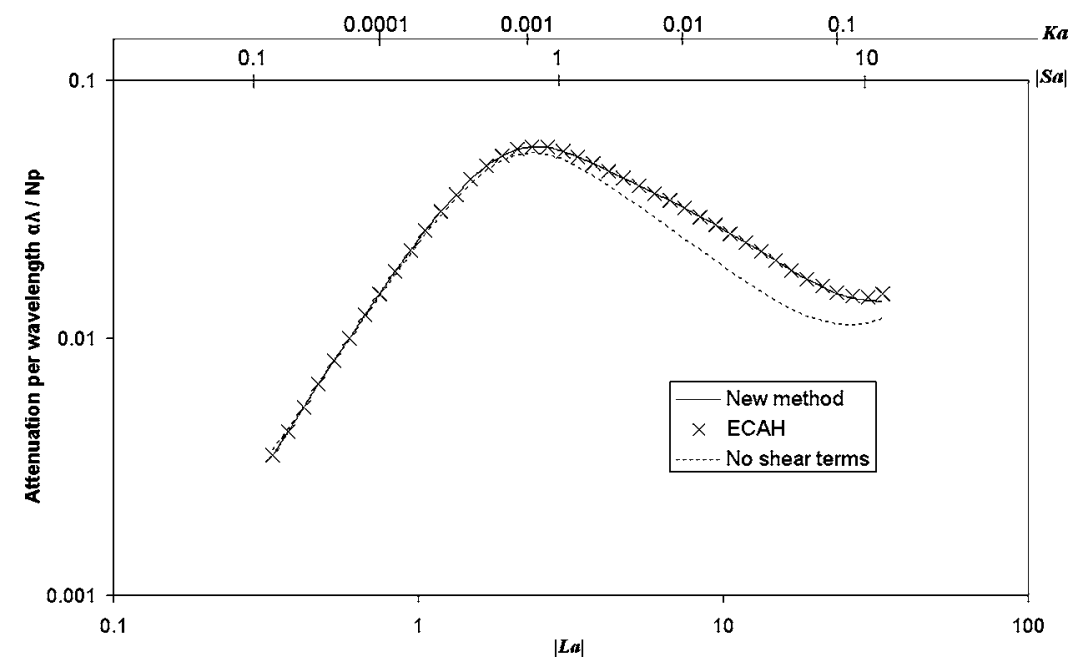

FIG. 2. Attenuation per wavelength as a function of the parameter $|L a|$ for $20 \%$ hexadecane in water (with $1 \%$ Tween20) at $20^{\circ} \mathrm{C}$ with a particle diameter of $1 \mu \mathrm{m}$. The new method is compared with the previous ECAH method (Epstein and Carhart, 1953) and in the absence of viscous/shear effects. The shear parameter $|\mathrm{Sa}|$ scales in proportion to $|L a|$ and is shown above the plot, along with the values of $K a$. The parameters for the dispersed phase are $\left|L^{\prime} a\right|=1.28|L a|,\left|S^{\prime} a\right|=0.52|S a|$, and $K^{\prime} a$ $=1.09 \mathrm{Ka}$. 


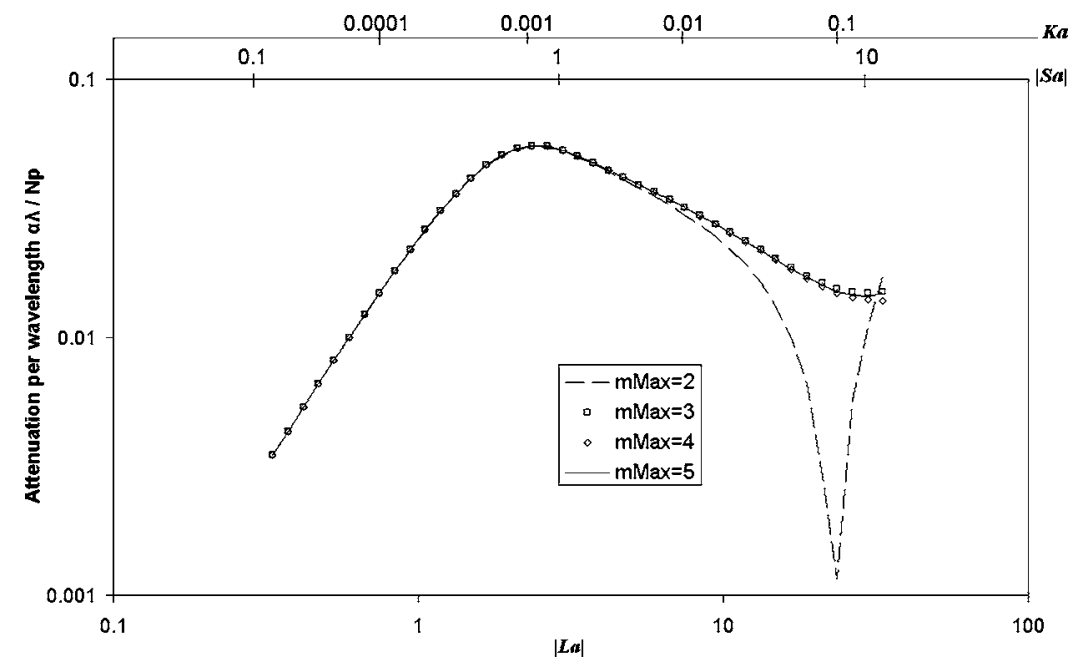

FIG. 3. Attenuation per wavelength as a function of the parameter $|L a|$ for $20 \%$ hexadecane in water (with $1 \%$ Tween20) at $20^{\circ} \mathrm{C}$ with a particle diameter of $1 \mu \mathrm{m}$. The plot shows the contribution of the first few orders in $m$, when the wave number is determined up to and including orders $n=2$. The shear parameter $|S a|$ scales in proportion to $|L a|$ and is shown above the plot, along with the values of $K a$. The parameters for the dispersed phase are $\left|L^{\prime} a\right|=1.28|L a|, \quad\left|S^{\prime} a\right|=0.52|S a|$, and $K^{\prime} a$ $=1.09 \mathrm{Ka}$.

Figures 1 and 2 show results calculated up to orders $n=2$ and $m=4$. These give good agreement with the ECAH method for $K a \leqslant 0.1$. As $K a$ increases, higher order terms are needed to obtain an accurate result. Figure 3 shows the contribution of including these terms. The solution up to order $m=2$, corresponding to the zero order (in $K a$ ) contribution to attenuation, is accurate for $K a \leqslant 0.007$. However, at larger values of $K a$, higher order terms must be included. The use of terms up to and including $m=4$, corresponding to terms in $(K a)^{2}$ in attenuation produces a good result over a much wider range of values of $K a$ (within the condition $K a \ll 1$ ).

\section{CONCLUSIONS}

The method presented here enables the scattering properties of a spherical object to be calculated in the longwavelength region, including the effects of heat fluctuations and viscous effects. The system configuration is identical to that solved by Epstein and Carhart (1953) and Allegra and Hawley (1972). However, the new method avoids the numerical problems associated with that well-established ECAH solution which uses Rayleigh expansions for the wave potentials and spherical Bessel and Hankel functions. These functions are difficult to calculate for large arguments and the matrix equation for the boundary conditions was numerically badly conditioned.

The results consist of a ladder of coefficients for each partial wave order, which when summed lead to the far-field scattering coefficient for that order. Recurrence relations [Eqs. (27) and (36)], followed by solution to the thermal boundary Eqs. (76) and (77) and then a matrix solution (81)-(83) produce coefficients which are then combined using Eq. (87) to obtain the far-field coefficient. This solution completes the application of Kleinman's method (Kleinman, 1965) to the acoustic scattering case, including thermal and shear effects. While this approach may appear more complicated than a direct solution to the boundary equations as in the ECAH method, it produces a numerically stable method, with no need for Bessel functions or a high-precision matrix inversion. The lowest partial wave orders can be obtained analytically as shown in Sec. V G and in fact in the longwavelength region only the lowest orders are significant. These solutions are equivalent to a series expansion in $\mathrm{Ka}$ of the analytical results obtained by ECAH in the longwavelength region. Although this has not been proven analytically, the numerical results demonstrate an accurate correspondence.

Although the results presented here can already be obtained by the ECAH solution, the new method was developed for several reasons. First, it was required to overcome the numerical difficulties with the ECAH solution, as has been stated. In addition, a more general method was sought to allow alternative particle shapes to be studied. At the current stage, the scattering potentials still use the partial wave expansions and a spherical particle shape, but future work may enable other shapes to be analyzed within this alternative approach. Kleinman (1965) developed his method in part to allow alternative shapes to be analyzed, without the requirement for special functions describing their geometry. His method was applied to single potential, whereas in the acoustical case propagational, thermal, and shear modes must be considered. A final intention of the development was to look at the problem of multiple scattering, having established a solution technique which would lend itself to the study of scattering at multiple centres. These items of study have yet to be achieved.

\section{ACKNOWLEDGMENT}

The research was supported by the U.K. Engineering and Physical Sciences Research Council (EPSRC), Grant GR/L/51034.

\section{APPENDIX}

For the incident field expansion in powers of $K a$ the function

$$
F_{n}(s)=\frac{2^{n}(2 n+1)(s+n) !}{s !(2 s+2 n+1) !}
$$

is used. In numerical calculations, the factorial functions suffer from overflow for all but very low orders $(n, s)$. The 
following recurrence relations can be used for accurate calculation:

$$
\begin{aligned}
& F_{0}(0)=1, \\
& \frac{F_{n}(0)}{F_{n-1}(0)}=\frac{1}{(2 n-1)} \text { for } n \geqslant 1, \\
& \frac{F_{n}(s)}{F_{n}(s-1)}=\frac{1}{2 s(2 n+2 s+1)} \text { for } s \geqslant 1 .
\end{aligned}
$$

The thermal and shear wave solutions use expansions of the spherical Bessel functions which are not generally found in mathematical texts. The coefficients can be calculated for the order $n$ by the formula below.

For the spherical Hankel function,

$$
\begin{aligned}
& h_{n}(x)=e^{i x} \sum_{j=1}^{n+1} \frac{h_{n j}}{x^{j}}, \\
& h_{01}=-i \text { for } n=0,
\end{aligned}
$$

for $n>0$

$$
h_{n, j}= \begin{cases}-i h_{n-1, j} & \text { for } j=1 \\ -i h_{n-1, j}+(n+j-2) h_{n-1, j-1} & \text { for } 1<j<n+1 \\ (2 n-1) h_{n-1, j-1} & \text { for } j=n+1 .\end{cases}
$$

Similarly, for the spherical Bessel function, this is defined in two parts, an outgoing and an ingoing traveling wave,

$j_{n}(x)=e^{i x} \sum_{l=1}^{n+1} \frac{j_{n l+}}{x^{l}}-e^{-i x} \sum_{l=1}^{n+1} \frac{j_{n l-}}{x^{l}}$,

$j_{0,1+}=1 / 2 \mathrm{i}$ for $n=0$,

for $n>0$

$j_{n, l+}= \begin{cases}-i j_{n-1, l+} & \text { for } l=1, \\ -i j_{n-1, l+}+(n+l-2) j_{n-1, l-1+} & \text { for } 1<l<n+1, \\ (2 n-1) j_{n-1, l-1+} & \text { for } l=n+1 .\end{cases}$ $j_{0,1-}=1 / 2 i \quad$ for $n=0$,

for $n>0$

$j_{n, l-}= \begin{cases}i j_{n-1, l-} & \text { for } l=1, \\ i j_{n-1, l-}+(n+l-2) j_{n-1, l-1-} & \text { for } 1<l<n+1, \\ (2 n-1) j_{n-1, l-1-} & \text { for } l=n+1 .\end{cases}$

Allegra, J. R., and Hawley, S. A. (1972). "Attenuation of sound in suspensions and emulsions: Theory and experiments," J. Acoust. Soc. Am. 51, $1545-1564$.

Challis, R. E., Povey, M. J. W., Mather, M. L., and Holmes, A. K. (2005).

"Ultrasound techniques for characterizing colloidal dispersions," Rep. Prog. Phys. 68, 1541-1637.

Colton, D., and Kress, R. (1988). Inverse Acoustic and Electromagnetic Scattering Theory, 2nd ed. (Springer, Berlin).

Epstein, P. S., and Carhart, R. R. (1953). "The absorption of sound in suspensions and emulsions. I. Water fog in air," J. Acoust. Soc. Am. 25, $553-565$.

Fikioris, J. G., and Waterman, P. C. (1964). "Multiple scattering of waves. II. 'Hole correction' in the scalar case," J. Math. Phys. 5, 1413-1420.

Harlen, O. G., Holmes, M. J., Povey, M. J. W., Qiu, Y., and Sleeman, B. D. (2001). "A low frequency potential scattering description of acoustic propagation in dispersions," SIAM J. Appl. Math. 61, 1906-1931.

Harlen, O. G., Holmes, M. J., Povey, M. J. W., and Sleeman, B. D. (2003).

"Acoustic propagation in dispersions and the geometric theory of diffraction,” SIAM J. Appl. Math. 63, 834-849.

Kleinman, R. E. (1965). "The Dirichlet problem for the Helmholtz equation," Arch. Ration. Mech. Anal. 18, 205-229.

Lloyd, P., and Berry, M. V. (1967). "Wave propagation through an assembly of spheres. IV. Relations between different multiple scattering theories," Proc. Phys. Soc. London 91, 678-688.

McClements, D. J., and Coupland, J. N. (1996). "Theory of droplet size distribution measurements in emulsions using ultrasonic spectroscopy," Colloids Surf., A 117, 161-170.

O'Neill, T. J., Tebbutt, J. S., and Challis, R. E. (2001). "Convergence criteria for scattering models of ultrasonic wave propagation in suspensions of particles," IEEE Trans. Ultrason. Ferroelectr. Freq. Control 48, 419-424.

Pinfield, V. J., Harlen, O. G., Povey, M. J. W., and Sleeman, B. D., (2006).

"Acoustic propagation in dispersions in the long wavelength limit," SIAM J. Appl. Math., 66, 489-509.

Povey, M. J. W. (1997). Ultrasonic Techniques for Fluids Characterization (Academic, San Diego).

Strutt, J. W. (Baron Rayleigh) (1896). The Theory of Sound, 2nd ed. (Macmillan, London).

Waterman, P. C., and Truell, R. (1961). "Multiple scattering of waves," J. Math. Phys. 2, 512-537. 